\title{
Stereoselective synthesis of cis-2-(polyfluoroalkyl)chroman-4-ols and trans-4-acylamino-2-(polyfluoroalkyl)chromans
}

\author{
Vyacheslav Ya. Sosnovskikh,* Roman A. Irgashev, and Anna A. Levchenko \\ Department of Chemistry, Ural State University, pr. Lenina 51, 620083 Ekaterinburg, Russian \\ Federation \\ E-mail: vyacheslav.sosnovskikh@usu.ru
}

Dedicated to Professor Alexander F. Pozharskii on his $70^{\text {th }}$ birthday

\begin{abstract}
trans-4-Acylamino-2-(polyfluoroalkyl)chromans were synthesized using a Ritter reaction between cis-2-(polyfluoroalkyl)chroman-4-ols and various nitriles.
\end{abstract}

Keywords: 2-(Polyfluoroalkyl)chromans, reduction, oxidation, dehydration, Ritter reaction

\section{Introduction}

The biological activity of many of the naturally occurring compounds which incorporate a chroman ring system has resulted in several applications of chromans of various levels of saturation and oxidation in synthesis. ${ }^{1}$ Furthermore, they have considerable biological importance, especially as potentially useful pesticides ${ }^{2}$ and drug candidates in the field of potassium channel openers. ${ }^{3}$ On the other hand, the polyfluoroalkyl groups are highly important substituents in the field of organic chemistry. The introduction of these groups into organic molecules can bring about some remarkable changes in the physical properties, chemical reactivity, and biological activity of the derived fluorinated compounds. ${ }^{4}$ In particular, due to the powerful electron-withdrawing ability of polyfluoroalkyl substituents $2-\mathrm{R}^{\mathrm{F}}$-chromones, ${ }^{5}$ $\mathrm{R}^{\mathrm{F}} \mathrm{CO}$-chromones ${ }^{6}$ and 3-nitro-2- $\mathrm{R}^{\mathrm{F}}$-chromenes ${ }^{7}$ have attracted our attention as highly reactive compounds, which can serve as the starting substances in the synthesis of a whole series of heterocycles with interesting properties.

However, to the best of our knowledge, very little is known about the synthesis and properties of partially fluorinated chroman derivatives. We have recently shown that the reaction of $2-\mathrm{R}^{\mathrm{F}}$-chromones with $\mathrm{CF}_{3} \mathrm{SiMe}_{3}$ proceeded as a 1,4-addition and provided an access to 2,2bis(polyfluoroalkyl)chroman-4-ones and their derivatives. ${ }^{8}$ Earlier 2,2-bis(trifluoromethyl)-6nitrochroman-4-one, which is a key structural element of the fluorinated analogues of an 
important class of potassium channel openers, benzopyran-4-amide derivatives, was prepared by treating 2-fluoro-5-nitroacetophenone with hexafluoroacetone trihydrate. ${ }^{9}$ Also, 2,2,6tris(trifluoromethyl)-2H-chromene, a key intermediate for the preparation of the fluorinated analogues of cromakalim, a highly potent antihypertensive drug, ${ }^{10}$ and 6,7-dimethoxy-2-methyl2-(trifluoromethyl)- $2 \mathrm{H}$-chromene, a trifluoromethyl analogue of the natural insect antijuvenile hormone precocene $\mathrm{II},{ }^{11}$ were described. There has been only one paper on the synthesis of chroman-4-one with a trifluoromethyl group at the 2 position. ${ }^{12}$ In view of the unique biological properties displayed by chromans ${ }^{13}$ on one hand and by many $\mathrm{CF}_{3}$-containing heterocycles ${ }^{4}$ on the other hand, it was of interest to obtain novel fluorine-containing chroman derivatives from 2$\mathrm{R}^{\mathrm{F}}$-chromones.

\section{Results and Discussion}

Chromones and chromanones can be reduced with different reagents but also undergo ring opening, hydrogenolysis and other side reactions which lower the yields of chroman-4-ols. ${ }^{14}$ Attempted reduction of chromones using hydride donors such as $\mathrm{NaBH}_{4}$ or hydrogenation using noble metal catalysts has been reported to lead to complex mixtures from over-reduction. ${ }^{15} \mathrm{We}$ found that $2-\mathrm{R}^{\mathrm{F}}$-chromones $1 \mathrm{a}-\mathrm{c}$ smoothly reacted with sodium borohydride in methanol to afford cis-2-(polyfluoroalkyl)chroman-4-ols $\mathbf{2 a - c}$ in good yields (65-83\%). The corresponding chromanone 3, intermediate of the reaction, is in situ reduced to chromanol 2 because the reduction of $\mathbf{3}$ is faster than that of $\mathbf{1}$ due to double bond conjugation in the latter. Reduction of $\mathbf{1}$ to $\mathbf{2}$ exhibits high stereoselectivity and the cis-structure of chromanols $\mathbf{2}$ is evident from the coupling constants. In the ${ }^{1} \mathrm{H}$ NMR spectra, the axial H-3 proton manifests itself at $\delta 2.03-2.10$ as a doublet of triplets or a doublet of doublets of doublets with the geminal constant ${ }^{2} J=12.8$ $13.4 \mathrm{~Hz}$ and the vicinal constants ${ }^{3} J_{\mathrm{H} 3, \mathrm{H} 2}=9.9-12.1 \mathrm{~Hz}$ and ${ }^{3} J_{\mathrm{H} 3, \mathrm{H} 4}=8.9-10.8 \mathrm{~Hz}$, which indicate the axial arrangement of the H-2 and $\mathrm{H}-4$ atoms. Therefore, chromanols 2 in $\mathrm{CDCl}_{3}$ are in the half-chair conformation with cis-diequatorial arrangement of the substituents. Initial attack at the carbonyl group of $\mathbf{3}$ by sodium borohydride might be expected to occur from the side opposite to the $\mathrm{R}^{\mathrm{F}}$ group leading to the cis-2. Note that reduction of flavones and chromones with $\mathrm{NaBH}_{4} / \mathrm{NiCl}_{2}$ in methanol also yields cis-flavan-4-ols and cis-chroman-4-ols. ${ }^{14}$

Selective reduction of the double bond in chromone 1a can be achieved by using of diisobutylaluminium hydride ( 1 equiv.) in THF-toluene solution at $-78{ }^{\circ} \mathrm{C}$. In this case, chroman-4-one 3a was obtained as a sole product in $56 \%$ yield. This is not surprising since reduction of isoflavones to isoflavan-4-ones under the same reaction conditions has been described previously. ${ }^{16}$ In addition, homoisoflavones has been converted into homoisoflavanones by subjecting to catalytic hydrogenation. ${ }^{17}$ The oxidation of chromanols $\mathbf{2 a , b}$ to chromanones 3a,b in $74-79 \%$ yields was performed with chromic acid (from sodium dichromate and sulfuric acid) in ethyl ether. ${ }^{18}$ Dehydration of chromanol 2a was effected by a trace of $p$-toluene sulfonic 
acid in boiling benzene for $5 \mathrm{~h}$ and subsequent distillation of the reaction mixture gave 2(trifluoromethyl)-2H-chromene 4 in 70\% yield, which was sensitive to oxygen of air.

Surprisingly, as in the case of 2-(trifluoromethyl)chroman-4-one 3a, whose isolation and characterization had not been reported prior to our work, ${ }^{12}$ none of these simple compounds was recorded in the literature. We regarded these compounds as desirable targets because of their relationship to naturally occurring benzopyran derivatives and usefulness as $\mathrm{R}^{\mathrm{F}}$-containing building blocks for the preparation of more complex partially fluorinated heterocycles.

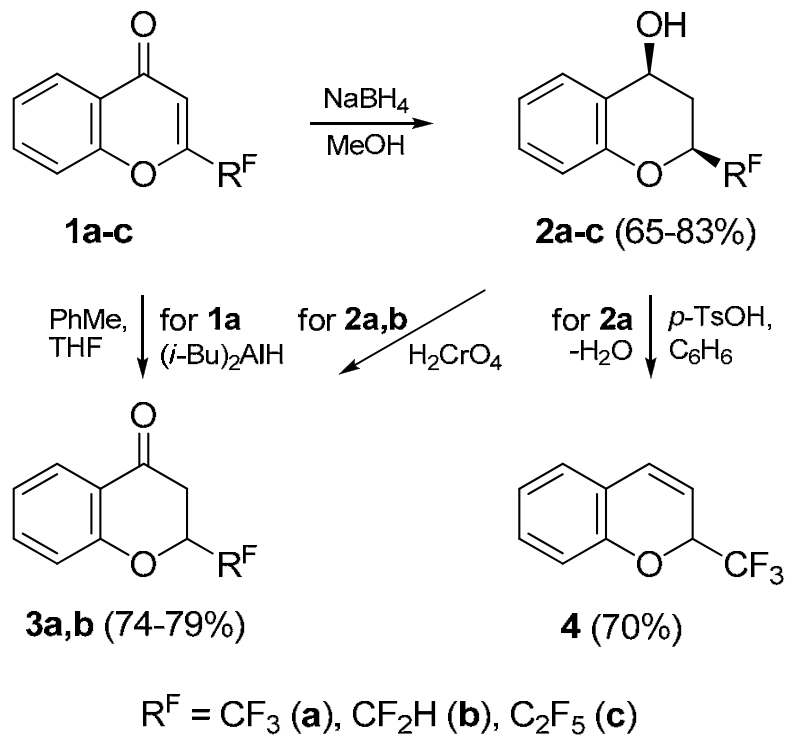

Scheme 1. Synthesis of $2-\mathrm{R}^{\mathrm{F}}$-chroman-4-ols $\mathbf{2 a}-\mathbf{c}, 2-\mathrm{R}^{\mathrm{F}}$-chroman-4-ones $\mathbf{3 a}, \mathbf{b}$ and $2-\mathrm{CF}_{3}-2 \mathrm{H}-$ chromene 4.

Application of the Ritter reaction conditions to 2-(polyfluoroalkyl)chroman-4-ols 2a-c gave the new compounds 4-acylamino-2-(polyfluoroalkyl)chromans 5a-f. This reaction proceeded smoothly for all substrates and analytically pure products can be isolated by simple filtration in excellent yields (ca. 95\%). The Ritter reaction was stereoselective and chromans 5a-f were obtained as mixtures of trans- and cis-isomers (trans/cis $=84 / 16-94 / 6$ ) without the formation of any side products. The determination of the isomers ratio can easily be performed by ${ }^{1} \mathrm{H}$ and ${ }^{19}$ F NMR spectroscopic analysis. The hydrolysis of 5a (trans/cis $=92 / 8$ ) as a representative example with concentrated hydrochloric acid led to the pure trans-4-amino-2(trifluoromethyl)chroman hydrochloride 6, which was converted into trans-4-benzamido-2(trifluoromethyl)chroman $\mathbf{5 f}$ under the action of sodium hydroxide and benzoyl chloride (Scheme 2).

The configurations of 5 were assigned from their ${ }^{1} \mathrm{H}$ NMR spectra, in which two sets of signals showed the presence of two diastereomers. In the major isomer, $J_{\mathrm{H} 2 \mathrm{a}, \mathrm{H} 3 \mathrm{a}}=10.8-12.5 \mathrm{~Hz}$ and $J_{\mathrm{H} 4 \mathrm{e}, \mathrm{H} 3 \mathrm{a}} \approx J_{\mathrm{H} 4 \mathrm{e}, \mathrm{H} 3 \mathrm{e}} \approx 3.7 \mathrm{~Hz}$; in the minor isomer, axial H-3 proton appeared as a quartet with $J_{\mathrm{H} 3 \mathrm{a}, \mathrm{H} 3 \mathrm{e}} \approx J_{\mathrm{H} 3 \mathrm{a}, \mathrm{H} 4 \mathrm{a}} \approx J_{\mathrm{H} 3 \mathrm{a}, \mathrm{H} 2 \mathrm{a}}=12.0-12.2 \mathrm{~Hz}$. The size of the coupling constants suggests a 2- 
equatorial-4-axial arrangement for the 2 and 4 substituents in the major (trans) isomer and a diequatorial arrangement in the minor (cis) isomer. Thus, in this series of chromans, cis-isomer is destabilized by repulsion between the 4-equatorial acylamino group and the peri-hydrogen. In the ${ }^{19} \mathrm{~F}$ NMR spectra of $\mathbf{5 a}, \mathbf{d}-\mathbf{f}$, the $\mathrm{CF}_{3}$ group of trans- and cis-isomers manifests itself as a doublet at $85.2-85.6$ and $84.3-84.7 \mathrm{ppm}$ with ${ }^{3} J_{\mathrm{F}, \mathrm{H}}=6.6-6.7$ and $6.4-6.5 \mathrm{~Hz}$, respectively.<smiles>[R]OC1C[C@H](O)c2ccccc2O1</smiles>

2a-c
$\underset{\mathrm{H}_{2} \mathrm{SO}_{4}}{\stackrel{\mathrm{RCN}}{\longrightarrow}}$<smiles>O=C[C@@H]1C[C@@H](P)Oc2ccccc21</smiles>

trans-5a-f<smiles>O=C[C@H]1CC([PH])Oc2ccccc21</smiles>

cis-5a-f<smiles></smiles><smiles>[NH3+][C@H]1C[C@H](C(F)(F)F)Oc2ccccc21</smiles>

trans-6 (85\%)<smiles>O=CN[C@@H]1C[C@H](C(F)(F)F)Oc2ccccc21</smiles>

trans-5f $(73 \%)$

\begin{tabular}{ccccc}
\hline $\mathbf{5}$ & $\mathrm{R}^{\mathrm{F}}$ & $\mathrm{R}$ & Ratio of trans/cis $\mathbf{5}$ & Yield (\%) \\
\hline $\mathbf{a}$ & $\mathrm{CF}_{3}$ & $\mathrm{Me}$ & $92: 8$ & 95 \\
$\mathbf{b}$ & $\mathrm{CF}_{2} \mathrm{H}$ & $\mathrm{Me}$ & $90: 10$ & 72 \\
$\mathbf{c}$ & $\mathrm{C}_{2} \mathrm{~F}_{5}$ & $\mathrm{Me}$ & $86: 14$ & 95 \\
$\mathbf{d}$ & $\mathrm{CF}_{3}$ & $\mathrm{CH}_{2} \mathrm{CO}_{2} \mathrm{Et}$ & $91: 9$ & 94 \\
$\mathbf{e}$ & $\mathrm{CF}_{3}$ & $\mathrm{CH}=\mathrm{CH}_{2}$ & $94: 6$ & 92 \\
$\mathbf{f}$ & $\mathrm{CF}_{3}$ & $\mathrm{Ph}$ & $94: 6$ & 97 \\
\hline
\end{tabular}

Scheme 2. Synthesis of 4-acylamino-2-(polyfluoroalkyl)chromans 5a-f.

This reaction is a simple and effective method for the preparation of protected 4-amino-2(polyfluoroalkyl)chromans 5. It should be noted that 4-amino-2,2-dimethyl-6-halogenochromans (non-fluorinated analogues of 5) are the key intermediates of the synthetic process, giving, in the last step, access to compounds structurally related to the potassium channel opener cromakalim. ${ }^{19}$ These 2,2-dimethylchromans are much more selective for insulin-secreting cells over vascular tissue than the reference molecule, cromakalim. ${ }^{19 \mathrm{c}}$ 


\section{Conclusions}

Reduction of 2-(polyfluoroalkyl)chromones with sodium borohydride and diisobutylaluminium hydride provides a simple and convenient preparative procedure from readily available starting materials to cis-2-(polyfluoroalkyl)chroman-4-ols and 2-(polyfluoroalkyl)chroman-4-ones, which may be considered as new precursors in the synthesis of other highly functionalized biologically and medicinally important products. Ritter reaction between cis-2(polyfluoroalkyl)chroman-4-ols and nitriles represents a stereoselective approach to the synthesis of a variety of trans-4-acylamino-2-(polyfluoroalkyl)chromans and trans-4-amino-2(polyfluoroalkyl)chromans.

\section{Experimental Section}

General Procedures. ${ }^{1} \mathrm{H}(400 \mathrm{MHz})$ and ${ }^{19} \mathrm{~F}(376 \mathrm{MHz})$ NMR spectra were recorded on a Bruker DRX-400 spectrometer in $\mathrm{CDCl}_{3}$ or DMSO- $d_{6}$ with TMS and $\mathrm{C}_{6} \mathrm{~F}_{6}$ as internal standards, respectively. IR spectra were recorded on a Perkin-Elmer Spectrum BX-II instrument as $\mathrm{KBr}$ discs. Elemental analyses were performed at the Microanalysis Services of the Institute of Organic Synthesis, Ural Branch, Russian Academy of Sciences. Melting points are uncorrected. All solvents used were dried and distilled as per standard procedures. The starting chromones 1a-c were prepared according to described procedure. ${ }^{20}$

\section{General procedure for the synthesis of cis-2-(polyfluoroalkyl)chroman-4-ols (2a-c)}

Sodium borohydride $(285 \mathrm{mg}, 7.5 \mathrm{mmol}$ ) was added to a cooled (with ice water) solution of chromone $1(5.0 \mathrm{mmol})$ in $5 \mathrm{ml}$ of methanol. The mixture was stirred at $5-10{ }^{\circ} \mathrm{C}$ for $0.5 \mathrm{~h}$ and diluted with water $(10 \mathrm{ml})$. The colorless crystals that formed were filtered, washed with water, dried, and recrystallized from hexane or heptane-ethyl ether (5:1).

cis-2-(Trifluoromethyl)chroman-4-ol (2a). Yield 83\%, mp 127-128 ${ }^{\circ} \mathrm{C}$. IR (KBr) 3462, 1613, 1581, 1488, $1462 \mathrm{~cm}^{-1}$; ${ }^{1} \mathrm{H}$ NMR $\left(400 \mathrm{MHz}, \mathrm{CDCl}_{3}\right) \delta 1.93$ (d, $1 \mathrm{H}, \mathrm{OH}, J=8.4 \mathrm{~Hz}$ ), 2.03 (ddd, $1 \mathrm{H}, \mathrm{H}-3 \mathrm{a}, J_{\mathrm{H} 3 \mathrm{a}, \mathrm{H} 3 \mathrm{e}}=12.9 \mathrm{~Hz}, J_{\mathrm{H} 3 \mathrm{a}, \mathrm{H} 2 \mathrm{a}}=12.1 \mathrm{~Hz}, J_{\mathrm{H} 3 \mathrm{a}, \mathrm{H} 4 \mathrm{a}}=10.8 \mathrm{~Hz}$ ), $2.53\left(\mathrm{ddd}, 1 \mathrm{H}, \mathrm{H}-3 \mathrm{e}, J_{\mathrm{H} 3 \mathrm{e}, \mathrm{H} 3 \mathrm{a}}\right.$ $\left.=12.9 \mathrm{~Hz}, J_{\mathrm{Hz}, \mathrm{H} 4 \mathrm{a}}=6.2 \mathrm{~Hz}, J_{\mathrm{H} 3 \mathrm{e}, \mathrm{H} 2 \mathrm{a}}=2.3 \mathrm{~Hz}\right), 4.50\left(\mathrm{dqd}, 1 \mathrm{H}, \mathrm{H}-2 \mathrm{a}, J_{\mathrm{H} 2 \mathrm{a}, \mathrm{H3} \mathrm{a}}=12.1 \mathrm{~Hz}, J_{\mathrm{H} 2 \mathrm{a}, \mathrm{CF} 3}=\right.$ $\left.6.0 \mathrm{~Hz}, J_{\mathrm{H} 2 \mathrm{a}, \mathrm{H} 3 \mathrm{e}}=2.3 \mathrm{~Hz}\right), 5.01(\mathrm{~m}, 1 \mathrm{H}, \mathrm{H}-4 \mathrm{a}), 6.91(\mathrm{dd}, 1 \mathrm{H}, \mathrm{H}-8, J=8.2,1.1 \mathrm{~Hz}), 7.03(\mathrm{td}, 1 \mathrm{H}$, $\mathrm{H}-6, J=7.5,1.1 \mathrm{~Hz}), 7.22$ (dddd, $\left.1 \mathrm{H}, \mathrm{H}-7, J=8.2,7.3,1.6 \mathrm{~Hz},{ }^{6} J_{\mathrm{H} 7, \mathrm{H} 4 \mathrm{a}}=0.7 \mathrm{~Hz}\right), 7.49(\mathrm{dt}, 1 \mathrm{H}$, $\mathrm{H}-5, J=7.7,1.2 \mathrm{~Hz}) ;{ }^{19} \mathrm{~F}$ NMR $\left(376 \mathrm{MHz}, \mathrm{CDCl}_{3}\right) \delta 82.82\left(\mathrm{~d}, \mathrm{CF}_{3}, J=6.0 \mathrm{~Hz}\right)$. Anal. Calcd for $\mathrm{C}_{10} \mathrm{H}_{9} \mathrm{~F}_{3} \mathrm{O}_{2}$ : C, 55.05; H, 4.16. Found: C, 54.96; H, 4.18.

cis-2-(Difluoromethyl)chroman-4-ol (2b). Yield 70\%, mp 79-80 ${ }^{\circ} \mathrm{C}$. IR (KBr) 3445, 1613, 1581, 1487, $1461 \mathrm{~cm}^{-1} ;{ }^{1} \mathrm{H}$ NMR (400 MHz, $\left.\mathrm{CDCl}_{3}\right) \delta 1.70(\mathrm{bs}, 1 \mathrm{H}, \mathrm{OH}), 2.05$ (dt, 1H, H-3a, $\left.J_{\mathrm{H} 3 \mathrm{a}, \mathrm{H} 3 \mathrm{e}}=13.4 \mathrm{~Hz}, J_{\mathrm{H} 3 \mathrm{a}, \mathrm{H} 2 \mathrm{a}}=J_{\mathrm{H3a}, \mathrm{H} 4 \mathrm{a}}=9.5 \mathrm{~Hz}\right), 2.43\left(\mathrm{ddd}, 1 \mathrm{H}, \mathrm{H}-3 \mathrm{e}, J_{\mathrm{H} 3 \mathrm{e}, \mathrm{H} 3 \mathrm{a}}=13.4 \mathrm{~Hz}, J_{\mathrm{H} 3 \mathrm{e}, \mathrm{H} 4 \mathrm{a}}=\right.$ $\left.5.7 \mathrm{~Hz}, J_{\mathrm{H} 3 \mathrm{e}, \mathrm{H} 2 \mathrm{a}}=3.0 \mathrm{~Hz}\right), 4.37\left(\mathrm{qdd}, 1 \mathrm{H}, \mathrm{H}-2 \mathrm{a}, J_{\mathrm{H} 2 \mathrm{a}, \mathrm{H} 3 \mathrm{a}}=J_{\mathrm{H} 2 \mathrm{a}, \mathrm{CF} 2}=9.9 \mathrm{~Hz}, J_{\mathrm{H} 2 \mathrm{a}, \mathrm{CHF} 2}=4.5 \mathrm{~Hz}\right.$, $\left.J_{\mathrm{H} 2 \mathrm{a}, \mathrm{H} 3 \mathrm{e}}=3.0 \mathrm{~Hz}\right), 4.98\left(\mathrm{dd}, 1 \mathrm{H}, \mathrm{H}-4 \mathrm{a}, J_{\mathrm{H} 4 \mathrm{a}, \mathrm{H} 3 \mathrm{a}}=8.9 \mathrm{~Hz}, J_{\mathrm{H} 4 \mathrm{a}, \mathrm{H} 3 \mathrm{e}}=5.7 \mathrm{~Hz}\right), 5.99\left(\mathrm{ddd}, 1 \mathrm{H}, \mathrm{CHF}_{2}\right.$, 
$\left.{ }^{2} J_{\mathrm{H}, \mathrm{F}}=55.2,55.9 \mathrm{~Hz}, J=4.5 \mathrm{~Hz}\right), 6.88(\mathrm{dd}, 1 \mathrm{H}, \mathrm{H}-8, J=8.2,1.2 \mathrm{~Hz}), 7.00(\mathrm{td}, 1 \mathrm{H}, \mathrm{H}-6, J=7.5$, $1.2 \mathrm{~Hz}$ ), 7.22 (dddd, $\left.1 \mathrm{H}, \mathrm{H}-7, J=8.2,7.3,1.7 \mathrm{~Hz},{ }^{6} J_{\mathrm{H} 7, \mathrm{H} 4 \mathrm{a}}=0.6 \mathrm{~Hz}\right), 7.43(\mathrm{ddd}, 1 \mathrm{H}, \mathrm{H}-5, J=7.8$, $\left.1.7 \mathrm{~Hz},{ }^{4} J_{\mathrm{H} 5, \mathrm{H} 4 \mathrm{a}}=1.0 \mathrm{~Hz}\right) ;{ }^{19} \mathrm{~F}$ NMR $\left(376 \mathrm{MHz}, \mathrm{CDCl}_{3}\right) \delta 30.63\left(\mathrm{ddd}, \mathrm{CFF},{ }^{2} J_{\mathrm{F}, \mathrm{F}}=291.4 \mathrm{~Hz}\right.$, $\left.{ }^{2} J_{\mathrm{F}, \mathrm{H}}=55.2 \mathrm{~Hz},{ }^{3} J_{\mathrm{F}, \mathrm{H}}=10.0 \mathrm{~Hz}\right), 34.97\left(\mathrm{ddd}, \mathrm{CFF},{ }^{2} J_{\mathrm{F}, \mathrm{F}}=291.4 \mathrm{~Hz},{ }^{2} J_{\mathrm{F}, \mathrm{H}}=55.9 \mathrm{~Hz},{ }^{3} J_{\mathrm{F}, \mathrm{H}}=9.8\right.$ $\mathrm{Hz}$ ). Anal. Calcd for $\mathrm{C}_{10} \mathrm{H}_{10} \mathrm{~F}_{2} \mathrm{O}_{2}$ : C, 60.00; H, 5.04. Found: C, 59.84; H, 4.90.

cis-2-(Perfluoroethyl)chroman-4-ol (2c). Yield 65\%, mp 120-121 ${ }^{\circ} \mathrm{C}$. IR (KBr) 3344, 3253, 1614, 1588, 1489, $1460 \mathrm{~cm}^{-1}$; ${ }^{1} \mathrm{H}$ NMR (400 MHz, $\left.\mathrm{CDCl}_{3}\right) \delta 1.90$ (d, 1H, OH, J=8.5 Hz), 2.10 $\left(\mathrm{td}, 1 \mathrm{H}, \mathrm{H}-3 \mathrm{a}, J_{\mathrm{H} 3 \mathrm{a}, \mathrm{H} 3 \mathrm{e}}=J_{\mathrm{H} 3 \mathrm{a}, \mathrm{H} 2 \mathrm{a}}=12.6 \mathrm{~Hz}, J_{\mathrm{H} 3 \mathrm{a}, \mathrm{H} 4 \mathrm{a}}=10.8 \mathrm{~Hz}\right), 2.55\left(\mathrm{ddt}, 1 \mathrm{H}, \mathrm{H}-3 \mathrm{e}, J_{\mathrm{H} 3 \mathrm{e}, \mathrm{H} 3 \mathrm{a}}=\right.$ $\left.12.8 \mathrm{~Hz}, J_{\mathrm{H} 3 \mathrm{e}, \mathrm{H} 4 \mathrm{a}}=6.2 \mathrm{~Hz}, J_{\mathrm{H} 3 \mathrm{e}, \mathrm{H} 2 \mathrm{a}}=J_{\mathrm{H} 3 \mathrm{e}, \mathrm{CFF}}=2.0 \mathrm{~Hz}\right), 4.62\left(\mathrm{dddd}, 1 \mathrm{H}, \mathrm{H}-2 \mathrm{a}, J_{\mathrm{H} 2 \mathrm{a}, \mathrm{CFF}}=17.0 \mathrm{~Hz}\right.$, $\left.J_{\mathrm{H} 2 \mathrm{a}, \mathrm{H} 3 \mathrm{a}}=12.0 \mathrm{~Hz}, J_{\mathrm{H} 2 \mathrm{a}, \mathrm{CFF}}=5.0 \mathrm{~Hz}, J_{\mathrm{H} 2 \mathrm{a}, \mathrm{H} 3 \mathrm{e}}=2.0 \mathrm{~Hz}\right), 5.04\left(\mathrm{dt}, 1 \mathrm{H}, \mathrm{H}-4 \mathrm{a}, J_{\mathrm{H} 4 \mathrm{a}, \mathrm{H} 3 \mathrm{a}}=10.0 \mathrm{~Hz}\right.$, $\left.J_{\mathrm{H} 4 \mathrm{a}, \mathrm{H} 3 \mathrm{e}} \approx J_{\mathrm{H} 4 \mathrm{a}, \mathrm{OH}}=7.5 \mathrm{~Hz}\right), 6.88(\mathrm{dd}, 1 \mathrm{H}, \mathrm{H}-8, J=8.2,1.0 \mathrm{~Hz}), 7.03(\mathrm{td}, 1 \mathrm{H}, \mathrm{H}-6, J=7.5,1.0$ $\mathrm{Hz}$ ), 7.22 (ddd, 1H, H-7, J=8.3, 7.3, $1.3 \mathrm{~Hz}$ ), 7.49 (dt, 1H, H-5, J= 7.7, 1.0 Hz). Anal. Calcd for $\mathrm{C}_{11} \mathrm{H}_{9} \mathrm{~F}_{5} \mathrm{O}_{2}$ : C, 49.26; $\mathrm{H}, 3.38$. Found: $\mathrm{C}, 49.00 ; \mathrm{H}, 3.28$.

2-(Trifluoromethyl)chroman-4-one (3a) from chromanol 2a. Ethyl ether $(350 \mathrm{ml})$ and chromanol 2a (35.6 g, $0.163 \mathrm{~mol})$ were placed in a round-bottom flask fitted with a stirrer, dropping funnel, and a condenser. The chromic acid solution, prepared from sodium dichromate dihydrate $(24.4 \mathrm{~g}, 0.082 \mathrm{~mol})$ and $20.0 \mathrm{ml}$ of concentrated sulfuric acid diluted to $120 \mathrm{ml}$, was added drop-wise to the stirred solution over $1 \mathrm{~h}$, maintaining the temperature at $5-10{ }^{\circ} \mathrm{C}$. The reaction mixture was stirred for $4 \mathrm{~h}$ at room temperature and allowed to stand overnight. Then the upper ether layer was separated, and the aqueous phase extracted with ether $(25 \mathrm{ml})$. The combined extracts were washed with a $5 \%$ solution of sodium carbonate $(2 \times 50 \mathrm{ml})$, then water $(50 \mathrm{ml})$, dried over anhydrous calcium chloride, and concentrated to afford the chromanone $3 \mathbf{a}$ as colorless crystals, $27.8 \mathrm{~g}$ (yield $79 \%$ ), crystallized from hexane, mp $85-86{ }^{\circ} \mathrm{C}$ (ref. ${ }^{12}$ : mp $84-85$ $\left.{ }^{\circ} \mathrm{C}\right)$. IR (KBr) 1704, 1614, 1604, 1578, 1475, $1464 \mathrm{~cm}^{-1} ;{ }^{1} \mathrm{H}$ NMR (400 MHz, $\left.\mathrm{CDCl}_{3}\right) \delta 2.92$ (dd, $1 \mathrm{H}, \mathrm{CHH}, J=16.9,3.9 \mathrm{~Hz}), 3.01(\mathrm{dd}, 1 \mathrm{H}, \mathrm{CHH}, J=16.9,12.3 \mathrm{~Hz}), 4.82(\mathrm{dqd}, 1 \mathrm{H}, \mathrm{CH}, J=12.3$, 5.8, 3.9 Hz), 7.09 (dd, 1H, H-8, J=8.4, $1.0 \mathrm{~Hz}$ ), 7.12 (ddd, 1H, H-6, J = 7.9, 7.3, $1.0 \mathrm{~Hz}$ ), 7.56 (ddd, 1H, H-7, $J=8.4,7.3,1.8 \mathrm{~Hz}), 7.91(\mathrm{dd}, 1 \mathrm{H}, \mathrm{H}-5, J=7.9,1.8 \mathrm{~Hz}) ;{ }^{19} \mathrm{~F}$ NMR $(376 \mathrm{MHz}$, $\left.\mathrm{CDCl}_{3}\right) \delta 83.83\left(\mathrm{~d}, \mathrm{CF}_{3}, J=5.8 \mathrm{~Hz}\right.$ ). Anal. Calcd for $\mathrm{C}_{10} \mathrm{H}_{7} \mathrm{~F}_{3} \mathrm{O}_{2}: \mathrm{C}, 55.56 ; \mathrm{H}, 3.26$. Found: C, 55.57; H, 3.12 .

2-(Trifluoromethyl)chroman-4-one (3a) from chromone 1a. To a solution of the chromone 1a $(1.5 \mathrm{~g}, 7.0 \mathrm{mmol})$ in dry tetrahydrofuran $(30 \mathrm{ml})$, a solution of DIBAL in toluene $(7.5 \mathrm{mmol})$ was added at $-78{ }^{\circ} \mathrm{C}$ under a nitrogen atmosphere. After $3 \mathrm{~h}$, the mixture was quenched with water $(5$ $\mathrm{ml}$ ) and allowed to warm to room temperature. After the addition of $\mathrm{KOH}$ ( $3 \mathrm{~g}$ in $5 \mathrm{ml}$ of water) and ether $(30 \mathrm{ml})$, the organic phase was separated, and the aqueous phase extracted with ether $(10 \mathrm{ml})$. The combined extracts were washed with saturated sodium chloride, dried, and evaporated to give chromanone 3a as colorless crystals, $850 \mathrm{mg}$ (yield 56\%), mp 85-86 ${ }^{\circ} \mathrm{C}$.

2-(Difluoromethyl)chroman-4-one (3b). This compound was prepared from chromanol $\mathbf{2 b}$ analogously to $\mathbf{3 a}$; yield $74 \%$ (5.9 g from $8.0 \mathrm{~g}$ chromanol $\mathbf{2 b}$ ), colorless crystals, $\mathrm{mp} 57-58{ }^{\circ} \mathrm{C}$ (heptane-ethyl ether). IR (KBr) 1698, 1606, 1577, 1474, $1463 \mathrm{~cm}^{-1} ;{ }^{1} \mathrm{H}$ NMR (400 MHz, $\left.\mathrm{CDCl}_{3}\right) \delta 2.83(\mathrm{dd}, 1 \mathrm{H}, \mathrm{CHH}, J=16.9,3.6 \mathrm{~Hz}), 2.94(\mathrm{dd}, 1 \mathrm{H}, \mathrm{CH} H, J=16.9,12.7 \mathrm{~Hz}), 4.82(\mathrm{tdt}$, 
$1 \mathrm{H}, \mathrm{CH}, J=12.7,7.8,3.5 \mathrm{~Hz}), 6.04\left(\mathrm{ddd}, 1 \mathrm{H}, \mathrm{CHF}_{2},{ }^{2} J_{\mathrm{H}, \mathrm{F}}=55.6,54.2 \mathrm{~Hz}, J=3.3 \mathrm{~Hz}\right), 7.04(\mathrm{dd}$, $1 \mathrm{H}, \mathrm{H}-8, J=8.4,1.1 \mathrm{~Hz}$ ), 7.08 (ddd, $1 \mathrm{H}, \mathrm{H}-6, J=7.9,7.3,1.1 \mathrm{~Hz}), 7.53$ (ddd, 1H, H-7, $J=8.4$, 7.3, $1.8 \mathrm{~Hz}), 7.90(\mathrm{dd}, 1 \mathrm{H}, \mathrm{H}-5, J=7.9,1.8 \mathrm{~Hz}) ;{ }^{19} \mathrm{~F}$ NMR $\left(376 \mathrm{MHz}, \mathrm{CDCl}_{3}\right) \delta 29.82$ (ddd, $\left.\mathrm{CFF},{ }^{2} J_{\mathrm{F}, \mathrm{F}}=294.9 \mathrm{~Hz},{ }^{2} J_{\mathrm{F}, \mathrm{H}}=55.6 \mathrm{~Hz},{ }^{3} J_{\mathrm{F}, \mathrm{H}}=12.7 \mathrm{~Hz}\right), 33.26\left(\mathrm{ddd}, \mathrm{CFF},{ }^{2} J_{\mathrm{F}, \mathrm{F}}=294.9 \mathrm{~Hz},{ }^{2} J_{\mathrm{F}, \mathrm{H}}\right.$ $=54.2 \mathrm{~Hz},{ }^{3} J_{\mathrm{F}, \mathrm{H}}=7.8 \mathrm{~Hz}$ ). Anal. Calcd for $\mathrm{C}_{10} \mathrm{H}_{8} \mathrm{~F}_{2} \mathrm{O}_{2}: \mathrm{C}, 60.61 ; \mathrm{H}, 4.07$. Found: $\mathrm{C}$, 60.60; H, 3.99 .

2-(Trifluoromethyl)-2H-chromene (4). A solution of chromanol 2a (5.5 g, $0.025 \mathrm{~mol})$ in benzene $(50 \mathrm{ml})$, along with a catalytic amount of $p$-toluene sulfonic acid, was refluxed for $5 \mathrm{~h}$ with a Dean-Stark trap under an atmosphere of nitrogen. After cooling, the reaction mixture was washed with a $40 \%$ solution of $\mathrm{KOH}$ and dried over $\mathrm{KOH}$ and the benzene was removed under vacuum. Distillation afforded $3.5 \mathrm{~g}$ (yield 70\%) of chromene 4 as a colorless oil, bp $80-81{ }^{\circ} \mathrm{C}$ at $15 \mathrm{~mm}$. IR (neat) 1650, 1608, 1488, $1459 \mathrm{~cm}^{-1}$; ${ }^{1} \mathrm{H}$ NMR (400 MHz, CDCl$\left.{ }_{3}\right) \delta 5.23$ (qdd, 1H, H$\left.2,{ }^{3} J_{\mathrm{H}, \mathrm{F}}=6.6 \mathrm{~Hz}, J=3.8,1.8 \mathrm{~Hz}\right), 5.65(\mathrm{dd}, 1 \mathrm{H}, \mathrm{H}-3, J=10.0,3.8 \mathrm{~Hz}), 6.66(\mathrm{dd}, 1 \mathrm{H}, \mathrm{H}-4, J=$ $10.0,1.2 \mathrm{~Hz}), 6.86$ (d, 1H, H-8, J=8.1 Hz), 6.91 (td, 1H, H-6, J = 7.4, $1.1 \mathrm{~Hz}), 7.01$ (dd, 1H, H$5, J=7.5,1.7 \mathrm{~Hz}), 7.16(\mathrm{td}, 1 \mathrm{H}, \mathrm{H}-7, J=7.6,1.7 \mathrm{~Hz}) ;{ }^{19} \mathrm{~F} \mathrm{NMR}\left(376 \mathrm{MHz}, \mathrm{CDCl}_{3}\right) \delta 82.04(\mathrm{~d}$, $\mathrm{CF}_{3}, J=6.6 \mathrm{~Hz}$ ). Anal. Calcd for $\mathrm{C}_{10} \mathrm{H}_{7} \mathrm{~F}_{3} \mathrm{O}: \mathrm{C}, 60.01 ; \mathrm{H}, 3.53$. Found: $\mathrm{C}, 59.75 ; \mathrm{H}, 3.32$.

\section{General procedure for the synthesis of compounds $5 \mathrm{a}-\mathrm{c}$}

A solution of chromanol $2(1.8 \mathrm{mmol})$ in $5 \mathrm{ml}$ of acetonitrile, containing $0.3 \mathrm{ml}$ of $98 \%$ sulfuric acid, was allowed to stand for 1 day at room temperature. Then the reaction mixture was poured into cold water $(40 \mathrm{ml})$ and the white precipitate collected by filtration, washed with water and dried.

4-Acetamido-2-(trifluoromethyl)chroman (5a). Yield 95\%, mp 181-182 ${ }^{\circ} \mathrm{C}$. IR (KBr) 3308 , 1648, 1587, 1527, 1489, $1459 \mathrm{~cm}^{-1} ;{ }^{1} \mathrm{H}$ NMR (400 MHz, DMSO-d $)$ trans-isomer $(92 \%) \delta 1.86$ (s, 3H, Me), 2.07-2.12 (m, 2H, CH 2$), 4.80$ (dqd, 1H, H-2, J = 12.5, 6.6, $1.5 \mathrm{~Hz}$ ), 5.07 (dt, 1H, H4, $J=7.9,3.8 \mathrm{~Hz}$ ), 6.96 (dd, 1H, H-8, $J=8.4,1.2 \mathrm{~Hz}$ ), 7.00 (td, 1H, H-6, J=7.6, $1.2 \mathrm{~Hz}$ ), 7.237.28 (m, 2H, H-5, H-7), $8.43(\mathrm{~d}, 1 \mathrm{H}, \mathrm{NH}, J=7.9 \mathrm{~Hz})$, cis-isomer $(8 \%) \delta 1.83(\mathrm{q}, 1 \mathrm{H}, \mathrm{CHH}, J=$ $12.0 \mathrm{~Hz}$ ), 1.93 (s, 3H, Me), 2.28 (ddd, 1H, CHH, J=12.5, 6.0, 2.0 Hz), 5.10-5.16 (m, 1H, H-2), 5.27-5.34 (m, 1H, H-4), 6.89 (dd, 1H, H-8, J = 8.2, 1.2 Hz), 6.99 (m, 1H, H-6), 7.16 (dt, 1H, H$5, J=7.7,1.2$ ), 7.20 (dddd, $1 \mathrm{H}, \mathrm{H}-7, J=8.2,7.3,1.7,0.9), 8.32(\mathrm{~d}, 1 \mathrm{H}, \mathrm{NH}, J=8.5 \mathrm{~Hz}) ;{ }^{19} \mathrm{~F}$ NMR (376 MHz, DMSO-d $)_{6}$ trans-isomer $(92 \%) \delta 85.51\left(\mathrm{~d}, \mathrm{CF}_{3},{ }^{3} J_{\mathrm{H}, \mathrm{F}}=6.6 \mathrm{~Hz}\right)$, cis-isomer $(8 \%) \delta 84.61\left(\mathrm{~d}, \mathrm{CF}_{3},{ }^{3} J_{\mathrm{H}, \mathrm{F}}=6.5 \mathrm{~Hz}\right)$. Anal. Calcd for $\mathrm{C}_{12} \mathrm{H}_{12} \mathrm{~F}_{3} \mathrm{NO}_{2}$ : C, 55.60; H, 4.67; N, 5.40. Found: C, 55.44; H, 4.51; N, 5.19.

4-Acetamido-2-(difluoromethyl)chroman (5b). Yield 72\%, mp 164-165 ${ }^{\circ} \mathrm{C}$. IR (KBr) 3317 , 1639, 1587, 1533, 1489, $1459 \mathrm{~cm}^{-1} ;{ }^{1} \mathrm{H}$ NMR (400 MHz, DMSO-d $)$ trans-isomer $(90 \%) \delta 1.84$ (s, 3H, Me), 1.90-2.01 (m, 2H, CH $), 4.41$ (qt, 1H, H-2, J=10.8, $3.5 \mathrm{~Hz}), 5.01$ (dt, 1H, H-4, J= $7.7,3.6 \mathrm{~Hz}), 6.32\left(\mathrm{td}, 1 \mathrm{H}, \mathrm{CF}_{2} \mathrm{H},{ }^{2} J_{\mathrm{H}, \mathrm{F}}=54.5 \mathrm{~Hz},{ }^{3} J_{\mathrm{H}, \mathrm{H}}=3.3 \mathrm{~Hz}\right), 6.90(\mathrm{dd}, 1 \mathrm{H}, \mathrm{H}-8, J=8.2,1.0$ $\mathrm{Hz}), 6.95$ (td, 1H, H-6, J=7.5, 1.1 Hz), 7.20-7.25 (m, 2H, H-5, H-7), 8.44 (d, 1H, NH, J=7.7 $\mathrm{Hz}$ ), cis-isomer $(10 \%) \delta 1.76(\mathrm{q}, 1 \mathrm{H}, \mathrm{CHH}, J=12.0 \mathrm{~Hz}), 1.93(\mathrm{~s}, 3 \mathrm{H}, \mathrm{Me}), 2.16$ (ddd, 1H, CHH, $J=12.7,5.8,1.8 \mathrm{~Hz}), 4.57-4.68(\mathrm{~m}, 1 \mathrm{H}, \mathrm{H}-2), 5.19-5.27(\mathrm{~m}, 1 \mathrm{H}, \mathrm{H}-4), 6.26\left(\mathrm{td}, 1 \mathrm{H}, \mathrm{CF}_{2} \mathrm{H},{ }^{2} J_{\mathrm{H}, \mathrm{F}}\right.$ 
$\left.=54.5 \mathrm{~Hz},{ }^{3} J_{\mathrm{H}, \mathrm{H}}=3.2 \mathrm{~Hz}\right), 6.84(\mathrm{dd}, 1 \mathrm{H}, \mathrm{H}-8, J=8.2,1.1 \mathrm{~Hz}), 6.94(\mathrm{td}, 1 \mathrm{H}, \mathrm{H}-6, J=7.6,1.1$ $\mathrm{Hz}$ ), 7.12-7.20 (m, 2H, H-5, H-7), 8.29 (d, 1H, NH, $J=8.5 \mathrm{~Hz}$ ); ${ }^{19} \mathrm{~F}$ NMR (376 MHz, DMSO$\left.d_{6}\right)$ trans-isomer $(90 \%) \delta 32.56\left(\mathrm{dd}, \mathrm{CF}_{2} \mathrm{H},{ }^{2} J_{\mathrm{F}, \mathrm{H}}=54.5 \mathrm{~Hz},{ }^{3} J_{\mathrm{F}, \mathrm{H}}=11.4 \mathrm{~Hz}\right)$, cis-isomer $(10 \%) \delta$ $31.72\left(\mathrm{dd}, \mathrm{CF}_{2} \mathrm{H},{ }^{2} J_{\mathrm{F}, \mathrm{H}}=54.5 \mathrm{~Hz},{ }^{3} J_{\mathrm{F}, \mathrm{H}}=11.4 \mathrm{~Hz}\right.$ ). Anal. Calcd for $\mathrm{C}_{12} \mathrm{H}_{13} \mathrm{~F}_{2} \mathrm{NO}_{2}$ : C, 59.75; H, 5.43; N, 5.81. Found: C, 59.88; H, 5.66; N, 5.80.

4-Acetamido-2-(perfluoroethyl)chroman (5c). Yield 95\%, mp 129-130 ${ }^{\circ} \mathrm{C}$. IR (KBr) 3310, 1650, 1588, 1527, 1489, $1458 \mathrm{~cm}^{-1} ;{ }^{1} \mathrm{H}$ NMR (400 MHz, DMSO-d $)$ trans-isomer $(86 \%) \delta 1.85$ (s, 3H, Me), 2.05-2.22 (m, 2H, $\left.\mathrm{CH}_{2}\right), 4.87-4.97$ (m, 1H, H-2), 5.12 (ddd, 1H, H-4, J = 7.7, 4.0, $2.7 \mathrm{~Hz}), 6.90(\mathrm{dd}, 1 \mathrm{H}, \mathrm{H}-8, J=8.3,1.1 \mathrm{~Hz}), 7.01$ (td, $1 \mathrm{H}, \mathrm{H}-6, J=7.5,1.1 \mathrm{~Hz}), 7.23-7.28$ (m, $2 \mathrm{H}, \mathrm{H}-5, \mathrm{H}-7), 8.48(\mathrm{~d}, 1 \mathrm{H}, \mathrm{NH}, J=7.9 \mathrm{~Hz})$, cis-isomer $(14 \%) \delta 1.90(\mathrm{q}, 1 \mathrm{H}, \mathrm{CHH}, J=12.0$ $\mathrm{Hz}), 1.94$ (s, 3H, Me), 2.25-2.31 (m, 1H, CHH), 5.23-5.39 (m, 2H, H-2, H-4), 6.84 (dd, 1H, H$8, J=8.2,1.0 \mathrm{~Hz}), 6.99$ (td, 1H, H-6, J=7.6, $1.0 \mathrm{~Hz}$ ), 7.15-7.22 (m, 2H, H-5, H-7), 8.33 (d, 1H, $\mathrm{NH}, J=8.6 \mathrm{~Hz}) ;{ }^{19} \mathrm{~F}$ NMR $\left(376 \mathrm{MHz}, \mathrm{DMSO}-d_{6}\right)$ trans-isomer $(86 \%) \delta 33.04(\mathrm{dd}, 1 \mathrm{~F}, \mathrm{CFF}$, $\left.{ }^{2} J_{\mathrm{F}, \mathrm{F}}=276.6 \mathrm{~Hz},{ }^{3} J_{\mathrm{F}, \mathrm{H}}=18.2 \mathrm{~Hz}\right), 39.40\left(\mathrm{dd}, 1 \mathrm{~F}, \mathrm{CFF},{ }^{2} J_{\mathrm{F}, \mathrm{F}}=276.6 \mathrm{~Hz},{ }^{3} J_{\mathrm{F}, \mathrm{H}}=5.1 \mathrm{~Hz}\right), 81.56(\mathrm{~s}$, $\left.3 \mathrm{~F}, \mathrm{CF}_{3}\right)$, cis-isomer $(14 \%) \delta 32.81\left(\mathrm{dd}, 1 \mathrm{~F}, \mathrm{CFF},{ }^{2} J_{\mathrm{F}, \mathrm{F}}=276.6 \mathrm{~Hz},{ }^{3} J_{\mathrm{F}, \mathrm{H}}=17.0 \mathrm{~Hz}\right), 39.07(\mathrm{dd}$, $\left.1 \mathrm{~F}, \mathrm{CFF},{ }^{2} J_{\mathrm{F}, \mathrm{F}}=276.6 \mathrm{~Hz},{ }^{3} J_{\mathrm{F}, \mathrm{H}}=5.1 \mathrm{~Hz}\right), 81.73\left(\mathrm{~s}, 3 \mathrm{~F}, \mathrm{CF}_{3}\right)$. Anal. Calcd for $\mathrm{C}_{13} \mathrm{H}_{12} \mathrm{~F}_{5} \mathrm{NO}_{2}$ : C, 50.49; H, 3.91; N, 4.53. Found: C, 50.59; H, 3.99; N, 4.40.

4-Carbethoxyacetamido-2-(trifluoromethyl)chroman (5d). This compound was prepared from chromanol 2a and ethyl cyanoacetate analogously to 5a-c. Yield 94\%, mp $138-139{ }^{\circ} \mathrm{C}$. IR (KBr) 3318, 1736, 1644, 1589, 1524, 1491, $1463 \mathrm{~cm}^{-1}$; ${ }^{1} \mathrm{H}$ NMR (400 MHz, DMSO-d $)$ transisomer $(91 \%) \delta 1.18(\mathrm{t}, 3 \mathrm{H}, \mathrm{Me}, J=7.1 \mathrm{~Hz}), 2.08-2.18\left(\mathrm{~m}, 2 \mathrm{H}, 3-\mathrm{CH}_{2}\right), 3.24$ (AB-system, 2H, $\left.\mathrm{CH}_{2}, J=15.2 \mathrm{~Hz}\right), 4.08\left(\mathrm{q}, 2 \mathrm{H}, \mathrm{OCH}_{2}, J=7.1 \mathrm{~Hz}\right), 4.74-4.83(\mathrm{~m}, 1 \mathrm{H}, \mathrm{H}-2), 5.06$ (dt, 1H, H-4, $J$ $=7.6,3.8 \mathrm{~Hz}), 6.97(\mathrm{~d}, 1 \mathrm{H}, \mathrm{H}-8, J=8.2 \mathrm{~Hz}), 7.02(\mathrm{td}, 1 \mathrm{H}, \mathrm{H}-6, J=7.5,1.1 \mathrm{~Hz}), 7.25-7.30(\mathrm{~m}$, $2 \mathrm{H}, \mathrm{H}-5, \mathrm{H}-7), 8.71(\mathrm{~d}, 1 \mathrm{H}, \mathrm{NH}, J=7.6 \mathrm{~Hz})$, cis-isomer $(9 \%) \delta 1.22(\mathrm{t}, 3 \mathrm{H}, \mathrm{Me}, J=7.1 \mathrm{~Hz})$, 1.85 (q, 1H, 3-CHH, $J=12.2 \mathrm{~Hz}$ ), 2.27-2.34 (m, 1H, 3-CHH), 3.33 (AB-system, 2H, $\mathrm{CH}_{2}, J=$ $15.2 \mathrm{~Hz}), 4.13$ (q, 2H, OCH $2, J=7.1 \mathrm{~Hz}), 5.10-5.20$ (m, 1H, H-2), 5.29-5.37 (m, 1H, H-4), 6.90 (dd, 1H, H-8, J = 8.2, 1.0 Hz), 6.98 (m, 1H, H-6), 7.19-7.26 (m, 2H, H-5, H-7), 8.62 (d, 1H, NH, $J=9.0 \mathrm{~Hz}) ;{ }^{19} \mathrm{~F}$ NMR $\left(376 \mathrm{MHz}, \mathrm{DMSO}-d_{6}\right)$ trans-isomer $(91 \%) \delta 85.18\left(\mathrm{~d}, \mathrm{CF}_{3},{ }^{3} J_{\mathrm{H}, \mathrm{F}}=6.6\right.$ $\mathrm{Hz}$ ), cis-isomer $(9 \%) \delta 84.29\left(\mathrm{~d}, \mathrm{CF}_{3},{ }^{3} J_{\mathrm{H}, \mathrm{F}}=6.4 \mathrm{~Hz}\right)$. Anal. Calcd for $\mathrm{C}_{15} \mathrm{H}_{16} \mathrm{~F}_{3} \mathrm{NO}_{4}: \mathrm{C}, 54.38 ; \mathrm{H}$, 4.87; N, 4.23. Found: C, 54.33; H, 4.54; N, 4.12.

4-Acrylamido-2-(trifluoromethyl)chroman (5e). This compound was prepared from chromanol 2a and acrylonitrile analogously to 5a-c. Yield 92\%, mp 180-181 ${ }^{\circ} \mathrm{C}$. IR (KBr) 3298, 1654, 1631, 1587, 1523, 1488, $1458 \mathrm{~cm}^{-1}$; ${ }^{1} \mathrm{H}$ NMR (400 MHz, DMSO-d $_{6}$ trans-isomer (94\%) $\delta$ 2.12-2.21 (m, 2H, $\mathrm{CH}_{2}$ ), 4.77-4.86 (m, 1H, H-2), 5.17 (dt, 1H, H-4, J = 7.7, 3.6 Hz), 5.66 (dd, $1 \mathrm{H},=\mathrm{CH}, J=8.0,4.3 \mathrm{~Hz}), 6.15-6.25\left(\mathrm{~m}, 2 \mathrm{H},=\mathrm{CH}_{2}\right), 6.98(\mathrm{dd}, 1 \mathrm{H}, \mathrm{H}-8, J=8.3,1.2 \mathrm{~Hz}), 7.01$ (td, 1H, H-6, J = 7.5, 1.2 Hz), 7.25-7.30 (m, 2H, H-5, H-7), 8.68 (d, 1H, NH, J = 7.7 Hz), cisisomer $(6 \%) \delta 1.89(\mathrm{q}, 1 \mathrm{H}, \mathrm{CHH}, J=12.2 \mathrm{~Hz}), 2.31-2.37(\mathrm{~m}, 1 \mathrm{H}, \mathrm{CHH}), 5.14-5.20(\mathrm{~m}, 1 \mathrm{H}, \mathrm{H}-$ 2), 5.37-5.45 (m, 1H, H-4), $5.70(\mathrm{dd}, 1 \mathrm{H},=\mathrm{CH}, J=9.8,2.8 \mathrm{~Hz}), 6.22-6.32\left(\mathrm{~m}, 2 \mathrm{H},=\mathrm{CH}_{2}\right), 6.91$ (dd, 1H, H-8, J = 8.3, 1.0 Hz), 6.98 (m, 1H, H-6), 7.11-7.24 (m, 2H, H-5, H-7), 8.60 (d, 1H, NH, $J=8.5 \mathrm{~Hz}) ;{ }^{19} \mathrm{~F}$ NMR $\left(376 \mathrm{MHz}, \mathrm{DMSO}-d_{6}\right)$ trans-isomer $(94 \%) \delta 85.51\left(\mathrm{~d}, \mathrm{CF}_{3},{ }^{3} J_{\mathrm{H}, \mathrm{F}}=6.6\right.$ 
$\mathrm{Hz})$, cis-isomer $(6 \%) \delta 84.65\left(\mathrm{~d}, \mathrm{CF}_{3},{ }^{3} J_{\mathrm{H}, \mathrm{F}}=6.5 \mathrm{~Hz}\right)$. Anal. Calcd for $\mathrm{C}_{13} \mathrm{H}_{12} \mathrm{~F}_{3} \mathrm{NO}_{2}: \mathrm{C}, 57.57 ; \mathrm{H}$, 4.46; N, 5.16. Found: C, 57.66; H, 4.39; N, 5.09.

trans-4-Amino-2-(trifluoromethyl)chroman hydrochloride (6). A suspension of amide 5a (300 $\mathrm{mg}, 1.16 \mathrm{mmol})$ in 36\% hydrochloric acid $(4 \mathrm{ml})$ and water $(2 \mathrm{ml})$ was refluxed for $7 \mathrm{~h}$ and allowed to stand overnight at room temperature. The solid product was collected by filtration, washed with ethyl ether and dried to give salt trans-6 as colorless needles, $250 \mathrm{mg}$ (yield 85\%), mp $240{ }^{\circ} \mathrm{C}$ (subl.). IR (KBr) 3408, 2890, 1613, 1598, 1586, 1515, 1491, $1463 \mathrm{~cm}^{-1} ;{ }^{1} \mathrm{H} \mathrm{NMR}$ $\left(400 \mathrm{MHz}, \mathrm{DMSO}-d_{6}\right) \delta 2.30(\mathrm{ddd}, 1 \mathrm{H}, \mathrm{CHH}, J=14.8,12.4,5.2), 2.54(\mathrm{dt}, 1 \mathrm{H}, \mathrm{CH}, J=14.8$, 2.2), 4.62 (bs, 1H, H-4), 5.20 (dqd, $1 \mathrm{H}, \mathrm{H}-2, J=12.4,6.4,2.2$ ), 7.01 (dd, $1 \mathrm{H}, \mathrm{H}-8, J=8.3,1.1$ Hz), 7.08 (td, 1H, H-6, J = 7.6, 1.1 Hz), 7.36 (ddd, 1H, H-7, J=8.3, 7.3, 1.7 Hz), 7.60 (dd, 1H, $\mathrm{H}-5, J=7.8,1.7), 8.90\left(\mathrm{bs}, 3 \mathrm{H}, \mathrm{N}^{+} \mathrm{H}_{3}\right) ;{ }^{19} \mathrm{~F} \mathrm{NMR}\left(376 \mathrm{MHz}, \mathrm{DMSO}-d_{6}\right) \delta 85.25\left(\mathrm{~d}, \mathrm{CF}_{3},{ }^{3} J_{\mathrm{H}, \mathrm{F}}=\right.$ 6.4 Hz). Anal. Calcd for $\mathrm{C}_{10} \mathrm{H}_{11} \mathrm{ClF}_{3} \mathrm{NO}$ : C, 47.35; H, 4.37; N, 5.52. Found: C, 47.36; H, 4.28; N, 5.38 .

trans-4-Benzamido-2-(trifluoromethyl)chroman (5f). Hydrochloride 6 (130 mg, $0.5 \mathrm{mmol})$ was treated with $3 \mathrm{ml}$ of $40 \%$ aqueous sodium hydroxide and the free base was extracted with ether $(2 \times 5 \mathrm{ml})$. The combined extracts were evaporated, the residue was dissolved in pyridine $(2 \mathrm{ml})$ and benzoyl chloride $(360 \mathrm{mg}, 2.6 \mathrm{mmol}$ ) was added. Then the solution was allowed to stand for 1 day at room temperature, poured into $10 \%$ hydrochloric acid $(10 \mathrm{ml})$ and the white precipitate collected by filtration, washed with water and dried to give trans-5f, $120 \mathrm{mg}$ (yield $73 \%$ ), $\mathrm{mp}>300{ }^{\circ} \mathrm{C}$ (decomp.). IR (KBr) 3320, 1630, 1579, 1514, $1487 \mathrm{~cm}^{-1} ;{ }^{1} \mathrm{H}$ NMR (400 MHz, DMSO-d $)_{6}$ trans-isomer (100\%) $\delta$ 2.20-2.25 (m, 2H, $\left.\mathrm{CH}_{2}\right), 5.00-5.06(\mathrm{~m}, 1 \mathrm{H}, \mathrm{H}-2), 5.37$ (dt, 1H, H-4, J = 7.9, 3.8 Hz), 6.98-7.03 (m, 2H, H-8, H-6) 7.25-7.32 (m, 2H, H-7, H-5), 7.447.49 (m, 2H, H-3', H-5'), 7.54 (tt, 1H, H-4', $J=7.3,1.3 \mathrm{~Hz}$ ), 7.88-7.92 (m, 2H, H-2', H-6'), 8.92 (d, $1 \mathrm{H}, \mathrm{NH}, J=7.9 \mathrm{~Hz}$ ). Anal. Calcd for $\mathrm{C}_{17} \mathrm{H}_{14} \mathrm{~F}_{3} \mathrm{NO}_{2}$ : C, 63.55; H, 4.39; N, 4.36. Found: $\mathrm{C}, 63.21 ; \mathrm{H}, 4.21 ; \mathrm{N}, 4.28$. In addition, this compound was prepared as a 94:6 mixture of transand cis-isomers from chromanol $\mathbf{2 a}$ and benzonitrile analogously to $\mathbf{5 a}-\mathbf{c} ;{ }^{19} \mathrm{~F}$ NMR (376 MHz, DMSO-d $)_{6}$ trans-isomer $(94 \%) \delta 85.62\left(\mathrm{~d}, \mathrm{CF}_{3},{ }^{3} J_{\mathrm{H}, \mathrm{F}}=6.7 \mathrm{~Hz}\right)$, cis-isomer $(6 \%) \delta 84.66\left(\mathrm{~d}, \mathrm{CF}_{3}\right.$, ${ }^{3} J_{\mathrm{H}, \mathrm{F}}=6.4 \mathrm{~Hz}$ ).

\section{Acknowledgements}

This work was financially supported by the RFBR (Grant 06-03-32388).

\section{References}

1. (a) Engler, T. A.; Reddy, J. P.; Combrink, K. D.; Velde, D. V. J. Org. Chem. 1990, 55, 1248. (b) Murugesh, M. G.; Subburaj, K.; Trivedi, G. K. Tetrahedron 1996, 52, 2217. (c) Engler, T. A.; LaTessa, K. O.; Iyengar, R.; Chai, W.; Agrios, K. Bioorg. Med. Chem. 1996, 4, 1755. (d) 
Mechoulam, R.; McCallum, N. K.; Burstein, S. Chem. Rev. 1976, 76, 75. (e) Lesch, B.; Toräng, J.; Nieger, M.; Bräse, S. Synthesis 2005, 1888.

2. (a) Bowers, W. S. In Comprehensive Insect Physiology, Biochemistry and Pharmacology; Gilbert, L. I., Kerkut, G. A. Eds.; Pergamon Press: Oxford, 1985; Vol. 8, p. 551. (b) Bowers, W. S.; Ohta, T.; Cleere, J. S.; Marsella, P. A. Science 1976, 193, 542.

3. (a) Bergmann R.; Gericke R. J. Med. Chem. 1990, 33, 492. (b) Burrell, G.; Cassidy, F.; Evans, J. M.; Lightowler, D.; Stemp, G. J. Med. Chem. 1990, 33, 3023. (c) Gericke R.; Harting, J.; Lues, I.; Schittenhelm, C. J. Med. Chem. 1991, 34, 3074.

4. Hiyama, T. Organofluorine Compounds. Chemistry and Application; Springer: Berlin, 2000.

5. Sosnovskikh, V. Ya. Russ. Chem. Rev. 2003, 72, 489. (b) Sosnovskikh, V. Ya.; Usachev, B. I.; Sizov, A. Yu.; Barabanov, M. A. Synthesis 2004, 942. (c) Sosnovskikh, V. Ya.; Usachev, B. I.; Sizov, A. Yu. Synlett 2004, 1765. (d) Sosnovskikh, V. Ya.; Usachev, B. I.; Sevenard, D. V.; Röschenthaler, G.-V. Tetrahedron 2003, 59, 2625. (e) Sosnovskikh, V. Ya.; Barabanov, M. A.; Usachev, B. I. Org. Lett. 2003, 5, 2501. (f) Sosnovskikh, V. Ya.; Usachev, B. I.; Sizov, A. Yu.; Vorontsov, I. I.; Shklyaev Yu. V. Org. Lett. 2003, 5, 3123.

6. (a) Sosnovskikh, V. Ya.; Irgashev, R. A.; Barabanov, M. A. Synthesis 2006, 2707. (b) Sosnovskikh, V. Ya.; Irgashev, R. A.; Kodess, M. I. Tetrahedron 2008, 64, 2997. (c) Sosnovskikh, V. Ya.; Moshkin, V. S.; Kodess, M. I. Tetrahedron 2008, 64, 7877. (d) Sosnovskikh, V. Ya.; Khalymbadzha, I. A.; Irgashev, R. A.; Slepukhin, P. A. Tetrahedron 2008, 64, 10172. (e) Sosnovskikh, V. Ya.; Irgashev, R. A. Lett. Org. Chem. 2007, 4, 344.

7. (a) Korotaev, V. Yu.; Sosnovskikh, V. Ya.; Kutyashev, I. B.; Kodess, M. I. Lett. Org. Chem. 2005, 2, 616. (b) Korotaev, V. Yu.; Sosnovskikh, V. Ya.; Kutyashev, I. B.; Kodess, M. I. Izv. Akad. Nauk, Ser. Khim. 2006, 309 (Russ. Chem. Bull., Int. Ed. 2006, 55, 317). (c) Korotaev, V. Yu.; Sosnovskikh, V. Ya.; Kutyashev, I. B.; Kodess, M. I. Izv. Akad. Nauk, Ser. Khim. 2006, 1945 (Russ. Chem. Bull., Int. Ed. 2006, 55, 2020). (d) Korotaev, V. Yu.; Sosnovskikh, V. Ya.; Kutyashev, I. B.; Barkov, A. Yu.; Shklyaev, Yu. V. Tetrahedron Lett. 2008, 49, 5376.

8. (a) Sosnovskikh, V. Ya.; Sevenard, D. V.; Usachev, B. I.; Röschenthaler, G.-V. Tetrahedron Lett. 2003, 44, 2097. (b) Sosnovskikh, V. Ya.; Usachev, B. I.; Sevenard, D. V.; Röschenthaler, G.-V. J. Org. Chem. 2003, 68, 7747. (c) Sosnovskikh, V. Ya.; Usachev, B. I.; Sevenard, D. V.; Röschenthaler, G.-V. J. Fluor. Chem. 2005, 126, 779.

9. Koga, H.; Sato, H.; Ishizawa, T.; Taka, N.; Takahashi, T. Tetrahedron Lett. 1995, 36, 87.

10. Fenwick, A.E. Tetrahedron Lett. 1993, 34, 1815.

11. Camps, F.; Coll, J.; Messeguer, A.; Pericás, M. A. J. Heterocycl. Chem. 1980, 17, 207.

12. Sevenard, D. V.; Sosnovskikh, V. Ya.; Kolomeitsev, A. A.; Königsmann, M. H.; Röschenthaler, G.-V. Tetrahedron Lett. 2003, 44, 7623.

13. Horton, D. A.; Bourne, G. T.; Smythe, M. L. Chem. Rev. 2003, 103, 893.

14. Khurana, J. M.; Chauhan, S. J. Chem. Res. (S) 2002, 201.

15. Sabui, S. K.; Mondal, P.; Venkateswaran, R. V. J. Chem. Res. (S) 2002, 428.

16. Antus, S.; Gottsegen, A.; Nógrádi, M. Synthesis 1981, 574. 
17. Rao, V. M.; Damu, G. L. V.; Sudhakar, D.; Siddaiah, V.; Rao, C. V. ARKIVOC 2008 (xi) 285.

18. Brown H. C., Garg, C. P. J. Am. Chem. Soc. 1961, 83, 2952.

19. (a) Khelili, S.; Nguyen, Q.-A.; Lebrun, P.; Delarge, J.; Pirotte, B. Pharm. Pharmacol. Commun. 1999, 5, 189. (b) Sebille, S.; Gall, D.; de Tullio, P.; Florence, X.; Lebrun, P.; Pirotte, B. J. Med. Chem. 2006, 49, 4690. (c) Sebille, S.; de Tullio, P.; Becker, B.; Antoine, M.-H.; Boverie, S.; Pirotte, B.; Lebrun, P. J. Med. Chem. 2005, 48, 614.

20. Sosnovskikh, V. Ya.; Usachev, B. I. Izv. Akad. Nauk, Ser. Khim. 2001, 434 [Russ. Chem. Bull., Int. Ed. 2001, 50, 453]. 